\title{
Model selection applied to reconstruction of the Primordial Power Spectrum
}

\author{
J. Alberto Vázquez ${ }^{a, b}$ M. Bridges ${ }^{a, b}$ M.P. Hobson ${ }^{b}$ A.N. Lasenby ${ }^{a, b}$ \\ ${ }^{a}$ Kavli Institute for Cosmology, Madingley Road, Cambridge CB3 0HA, UK. \\ ${ }^{b}$ Astrophysics Group, Cavendish Laboratory, JJ Thomson Avenue, Cambridge CB3 0HE, \\ UK. \\ E-mail: jv292@cam.ac.uk, mb435@mrao.cam.ac.uk,mph@mrao.cam.ac.uk, \\ a.n.lasenby@mrao.cam.ac.uk
}

\begin{abstract}
The preferred shape for the primordial spectrum of curvature perturbations is determined by performing a Bayesian model selection analysis of cosmological observations. We first reconstruct the spectrum modelled as piecewise linear in $\log k$ between nodes in $k$-space whose amplitudes and positions are allowed to vary. The number of nodes together with their positions are chosen by the Bayesian evidence, so that we can both determine the complexity supported by the data and locate any features present in the spectrum. In addition to the node-based reconstruction, we consider a set of parameterised models for the primordial spectrum: the standard power-law parameterisation, the spectrum produced from the Lasenby \& Doran (LD) model and a simple variant parameterisation. By comparing the Bayesian evidence for different classes of spectra, we find the power-law parameterisation is significantly disfavoured by current cosmological observations, which show a preference for the LD model.
\end{abstract}

Keywords: Primordial Power Spectrum, Cosmological Parameters from CMBR, Inflation, Bayesian Analysis

ArXiv ePrint: 1203.1252 


\section{Contents}

1 Introduction 1

2 Bayesian Inference $\quad 2$

2.1 Parameter Estimation 2

2.2 Model Selection 3

3 Primordial Power Spectrum Parameterisation 4

3.1 Power spectrum reconstruction I 4

$\begin{array}{lll}3.2 & \text { Power spectrum reconstruction II } & 7\end{array}$

$\begin{array}{lll}3.2 .1 & \text { Running-like spectrum } & 7\end{array}$

$\begin{array}{ll}3.2 .2 & \text { Cut-off and Broken spectra }\end{array}$

$\begin{array}{lll}3.3 & \text { Power-law and running spectra } & 10\end{array}$

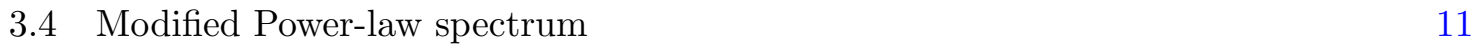

3.5 Lasenby \& Doran spectrum 12

4 Discussion and Conclusions $\quad 14$

$\begin{array}{lr}\text { A Cubic spline interpolated spectrum } & 18\end{array}$

\section{Introduction}

Cosmological inflation predicts the initial power spectrum of scalar density fluctuations to be close to scale-invariant with just a slight scale dependence. The simplest proposal for the shape of the spectrum is to assume a power-law parameterisation in terms of a spectral amplitude $A_{\mathrm{s}}$ and a spectral index or tilt parameter $n_{\mathrm{s}}$. Although this form has been in good agreement with cosmological observations, recent analyses from the Wilkinson Microwave Anisotropy Probe satellite (WMAP; [1]), and the Atacama Cosmology Telescope (ACT; $[2])$ have confirmed that the scale invariant $\left(n_{\mathrm{s}}=1\right)$ spectrum is now excluded at $3 \sigma$ C.L. Similar results are obtained when measurements from the South Pole Telescope (SPT; [3]) in combination with the WMAP data are considered. It has also been shown that if a running of the spectral index is taken into account, allowing deviations from the power-law spectrum, WMAP + ACT and WMAP+ SPT data show a preference for a negative running value at $1.8 \sigma$ C.L. Thus, consideration of models that slightly deviate from the simple power-law might be required. There have been several alternatives proposed. Some physically motivated models include an exponential large scale cut-off [4], discontinuities in the early universe from phase transitions [5], closed universe inflation [6], models in which the power spectrum drops to zero below some cut-off scale [7]. Some use observational data to constrain an a priori parameterisation or attempt a direct reconstruction using wavelets [8], deconvolution methods [9-11], binning the spectrum into an arbitrary number of band powers [12-15], Bayesian reconstruction [16], principle component analysis [17] and minimising the level of complexity needed via a cross-validation [18], amongst many others.

In this paper, we are interested in selecting the preferred shape for the primordial spectrum using the Bayesian evidence as an implementation of Occam's razor: a simpler model 
should be preferred, unless the data require a more sophisticated model. First, we determine the structure of the primordial power spectrum using an optimal model-free reconstruction. Our approach considers possible deviations from the power-law parameterisation by modelling the spectrum as a linear interpolation between a set of 'nodes' which can vary in both amplitude and $k$-position. Within this analysis we have included phenomenological features which might be relevant to the description of CMB observations, such as a large scale cut-off, a broken spectrum and a spectrum with a possible turn-over at any position in $k$-space. The reconstruction process is essentially the same binning format used previously by a number of authors, however here we allow the data to decide the level of complexity of the model - the number of nodes and their optimum position - via the Bayesian evidence. Then, for comparison, we compute the Bayesian evidence for a set of existing model proposals: a power-law parameterisation including both tilt and running parameter, a modified power-law spectrum to account for a drop off at large scales and the Lasenby \& Doran (LD) model based on a closed universe. Finally, for each model we compare its Bayesian evidence and according to the Jeffreys guideline we select the best model preferred by current data. In a previous paper [19], we have constrained the parameter space which describes the primordial spectrum derived from the LD model. In this work, using an optimal model-free reconstruction, the shape of the spectrum is determined directly from the data.

The paper is organised as follows: in the next Section we describe the basic parameter estimation and model selection, we also list the datasets and the cosmological parameters considered. In Section 3 we carry out the reconstruction for the primordial power spectrum and also consider different existing parameterisations suggested to describe the form of the spectrum. We show the resulting parameter constraints and the evidence for each worked model. Finally, in Section 4 we decide which model provides the best description for current observational data and present our conclusions.

\section{Bayesian Inference}

\subsection{Parameter Estimation}

Given a model or hypothesis $H$ for some data $\mathbf{D}$, Bayes' theorem tells us how to determine the probability distribution of the set of parameters $\Theta$ on which the model depends. Bayes' theorem states that

$$
\operatorname{Pr}(\boldsymbol{\Theta} \mid \mathbf{D}, H)=\frac{\operatorname{Pr}(\mathbf{D} \mid \boldsymbol{\Theta}, H) \operatorname{Pr}(\boldsymbol{\Theta} \mid H)}{\operatorname{Pr}(\mathbf{D} \mid H)},
$$

where, for brevity, we denote $\operatorname{Pr}(\boldsymbol{\Theta} \mid H) \equiv \pi(\boldsymbol{\Theta})$ as the prior probability which is upgraded through the likelihood $\operatorname{Pr}(\mathbf{D} \mid \boldsymbol{\Theta}, H) \equiv \mathcal{L}(\boldsymbol{\Theta})$ when experimental data $\mathbf{D}$ are considered. The aim for parameter estimation is to obtain the posterior probability $\operatorname{Pr}(\boldsymbol{\Theta} \mid \mathbf{D}, H)$ which represents the state of knowledge once we have taken into account the new information. The normalisation constant is usually refered to the Bayesian evidence $\operatorname{Pr}(\mathbf{D} \mid H) \equiv \mathcal{Z}$. Since this quantity is independent of the parameters $\boldsymbol{\Theta}$, it is often ignored in parameter estimation but plays the central role for model selection.

Throughout the analysis we compute posterior probabilities for each model in the light of temperature and polarisation measurements from the 7-year data release of the Wilkinson 
Microwave Anisotropy Probe (WMAP; [1]). The ACT and SPT data provide similar constraints on the primary cosmological parameters [2,3], thus since our results are not likely to be significantly affected according to which is chosen, and to avoid possible problems due to overlap of the sky regions, we have restricted the current analysis to use of just the ACT data. In addition to CMB data, we include distance measurements from the Supernova Cosmology Project Union 2 compilation (SCP; $[20]$ ) and large scale structure data from the Sloan Digital Sky Survey (SDSS) Data Release 7 (DR7) Luminous Red Galaxy (LRG) power spectrum [21]. We also impose a Gaussian prior from measurements of the Hubble parameter today $H_{0}$ from the Hubble Space Telescope (HST; [22]) key project.

We consider purely Gaussian adiabatic scalar perturbations and neglect tensor contributions. We assume a flat $\Lambda \mathrm{CDM}$ model ${ }^{1}$ specified by the following parameters: the physical baryon $\Omega_{\mathrm{b}} h^{2}$ and cold dark matter density $\Omega_{\mathrm{DM}} h^{2}$ relative to the critical density ( $h$ is the dimensionless Hubble parameter such that $H_{0}=100 h \mathrm{kms}^{-1} \mathrm{Mpc}^{-1}$ ), $\theta$ is $100 \times$ the ratio of the sound horizon to angular diameter distance at last scattering surface and $\tau$ denotes the optical depth at reionisation. Aside from the Sunyaev-Zel'dovich (SZ) amplitude $A_{S Z}$ used by WMAP analyses, the $148 \mathrm{GHz}$ ACT likelihood incorporates two additional secondary parameters: the total Poisson power $A_{p}$ at $l=3000$ and the amplitude of the clustered power $A_{c}$. The parameters describing the primordial spectra in each model are listed in Section 3, together with their flat priors imposed in our Bayesian analysis.

\subsection{Model Selection}

Model selection applies a similar type of analysis to parameter estimation but now at the level of models rather than parameters. The key quantity to bear in mind is the Bayesian evidence. It balances the complexity of cosmological models and then, naturally, incorporates Occam's razor. Let us consider several models or hypotheses $H_{i}$, each of them with prior probability $\operatorname{Pr}\left(H_{i}\right)$. Bayes' theorem for model selection is

$$
\frac{\operatorname{Pr}\left(H_{i} \mid \mathbf{D}\right)}{\operatorname{Pr}\left(H_{j} \mid \mathbf{D}\right)}=\frac{\operatorname{Pr}\left(\mathbf{D} \mid H_{i}\right)}{\operatorname{Pr}\left(\mathbf{D} \mid H_{j}\right)} \frac{\operatorname{Pr}\left(H_{i}\right)}{\operatorname{Pr}\left(H_{j}\right)} .
$$

The left-hand side denotes the relative probability of the model given the data, whereas in the right-hand side appears the key quantity to compute: the Bayesian evidence for each model. This evidence is nothing more than the average of the likelihood over the prior

$$
\mathcal{Z}=\int \mathcal{L}(\Theta) \pi(\Theta) d^{N} \Theta
$$

where $N$ is the dimensionality of the parameter space. Note that a complex model will usually lead to a higher likelihood, but if the fit is nearly as good as a simple model, the evidence will favour the simpler model through the smaller prior volume.

For convenience, the ratio of two evidences $\mathcal{Z}_{0} / \mathcal{Z}_{1}$ (or equivalently the difference in log evidences $\ln \mathcal{Z}_{0}-\ln \mathcal{Z}_{1}$ ) is often termed the Bayes factor $\mathcal{B}_{0,1}$ :

$$
\mathcal{B}_{0,1}=\ln \frac{\mathcal{Z}_{0}}{\mathcal{Z}_{1}}
$$

\footnotetext{
${ }^{1}$ Except for the LD model, which is based on a closed universe $\Omega_{k}<0$.
} 
Table 1. Jeffreys guideline scale for evaluating the strength of evidence when two models are compared.

\begin{tabular}{cccc}
\hline$\left|\mathcal{B}_{0,1}\right|$ & Odds & Probability & Strength \\
\hline$<1.0$ & $<3: 1$ & $<0.750$ & Inconclusive \\
$1.0-2.5$ & $\sim 12: 1$ & 0.923 & Significant \\
$2.5-5.0$ & $\sim 150: 1$ & 0.993 & Strong \\
$>5.0$ & $>150: 1$ & $>0.993$ & Decisive \\
\hline \hline
\end{tabular}

Then, the quantity $\mathcal{B}_{0,1}$ provides an idea on how well model 0 may fit the data when is compared to model 1. Jeffreys provided a suitable guideline scale on which we are able to make qualitative conclusions (see Table 1). In this work, we refer to positive (negative) values of $\mathcal{B}_{0,1}$ as the 0 model being favoured (disfavoured) over model 1.

The computation of the integral in Equation (2.3) is a very computationally demanding process, since it requires a multidimensional integration over the likelihood and prior. To carry out the exploration of the cosmological parameter space we first modify the CAMB code [23] in order to input an arbitrary shape of the primordial power spectrum ${ }^{2}$, then we incorporate into the CosmoMC software [24] a substantially improved and fully-parallelized version of the nested sampling algorithm called MultiNest [25, 26]. The MultiNest algorithm increases the sampling efficiency for calculating the evidence and allows one to obtain posterior samples even from distributions with multiple modes and/or pronounced degeneracies between parameters.

\section{Primordial Power Spectrum Parameterisation}

\subsection{Power spectrum reconstruction I}

First, we perform a reconstruction for the shape of the primordial spectrum. We parameterise $\mathcal{P}_{\mathcal{R}}(k)$ with a specific number of bins, logarithmically spaced in $k$, and varying only each amplitude, denoted $A_{\mathrm{s}, k_{i}}$. Throughout, we assume that most of the current relevant information is encompassed within the scales $k_{\min }=0.0001 \mathrm{Mpc}^{-1}$ and $k_{\max }=0.3 \mathrm{Mpc}^{-1}$, where the combined WMAP+ACT data significantly improves the parameter constraints. Outside of these boundaries we take the spectrum to be constant with values equal to those at $k_{\min }$ and $k_{\max }$ respectively. We allow variations in the spectral amplitudes with a conservative prior $A_{\mathrm{s}, k_{i}} \in[1,50] \times 10^{-10}$.

To maintain continuity between $k$-nodes, a linear interpolation is performed such that the

\footnotetext{
${ }^{2}$ A modified CAMB code version which allows a Node-based Parameterisation for the primordial power spectrum is a available at http://www.mrao.cam.ac.uk/software/
} 
form of the power spectrum is described by

$$
\begin{aligned}
& \qquad \mathcal{P}_{\mathcal{R}}(k)=\left\{\begin{array}{cl}
A_{\mathrm{s}, k_{\min }} & k \leq k_{\min } \\
A_{\mathrm{s}, k_{i}} & k \in\left\{k_{i}\right\} \\
A_{\mathrm{s}, k_{\max }} & k \geq k_{\max }
\end{array}\right. \\
& \text { and with linear interpolation for } \quad k_{\min } \leq k_{i}<k<k_{i+1} \leq k_{\max } .
\end{aligned}
$$

We start our reconstruction by considering the base model is equivalent to the HarrisonZel'dovich (HZ) spectrum $\left(n_{\mathrm{s}}=1\right)$ where the spectral amplitude $\mathcal{P}_{\mathcal{R}}(k)=A_{\mathrm{s}}$ is the only parameter, see Figure 1 . The next model, (b), allows for two amplitudes located at $k_{\min }$ and $k_{\max }$ to vary independently, thus emulating a tilted spectrum. We then add a third point (c) placed midway between the two existing nodes in (b). This model mimics a degree of spectral running by allowing slight variations in the interpolated slopes between the three nodes. Since these amplitudes are independent of each other, however, there is no need to pick any particular pivot point as in the case of power-law parameterisation, hence providing more freedom in the shape of the spectrum. The presence of a turn-over in the global structure of the resultant spectrum, shown in Figure 1 (c), points to some deviation from a simple tilt. We might continue adding nodes in this fashion until some arbitrary accuracy of model fit is achieved, but always bearing in mind that the inclusion of new unnecessary nodes is penalised through the Bayesian evidence. We, then consider a fourth stage where the $k$-space is logarithmically split into three equally spaced regions, (d). At small scales the shape of the power spectrum is well constrained with tight error bars on each node, whereas on large scales the error bars tell us there is still room for new features (within the limited amount of information due to cosmic variance). Notice the increased error bars due to the addition of an arbitrary number of band-powers and correlations created between them. We also observe the evidence has dropped for four $k$-nodes, therefore this stage seems to be a reasonable point to stop adding parameters in the reconstruction process. Figure 1 illustrates the corresponding form of the reconstructed spectra from the mean posterior estimates (with $1 \sigma$ error bars on its corresponding amplitudes), together with 1D marginalised posterior distribution for the amplitude at each node and for each reconstruction. In each model we include the Bayes factor compared to the base model (HZ).

The reconstructed spectra are assessed according to the Jeffreys guideline shown in Table 1. The Bayesian evidence between the base model and the two-node model $\mathcal{B}_{2,1}=+2.93 \pm 0.30$ points out that the $\mathrm{HZ}$ is strongly disfavoured when compared to a tilted spectrum, in agreement with WMAP/ACT [2] results. The addition of complexity in the third stage provides more flexibility in the shape of the reconstructed spectrum. The evidence between the twonode and three-node model, $\mathcal{B}_{2,3}=+0.18 \pm 0.30$, is too small to draw any decisive conclusion, though the evidence marginally prefers the simple tilted spectrum. Although the reconstructed shape of the spectrum in the fourth stage is similar to the one obtained in the second stage, the four $k$-node model is penalised because of the inclusion of unnecessary information. Thus, the peak of the evidence at model (b) shows that the optimal reconstruction contains, surprisingly, just two nodes, as is shown in Figure 1. Therefore, according to this reconstruction process, parameterisations such as the $\mathrm{HZ}$ and those containing more than three $k$-nodes are hence disfavoured by current observations. At this point of the analysis, with fixed $k$-node positions, our results are consistent with those obtained by [15], where accord- 
(a) $\mathcal{B}_{1,1}=0.00 \pm 0.30$
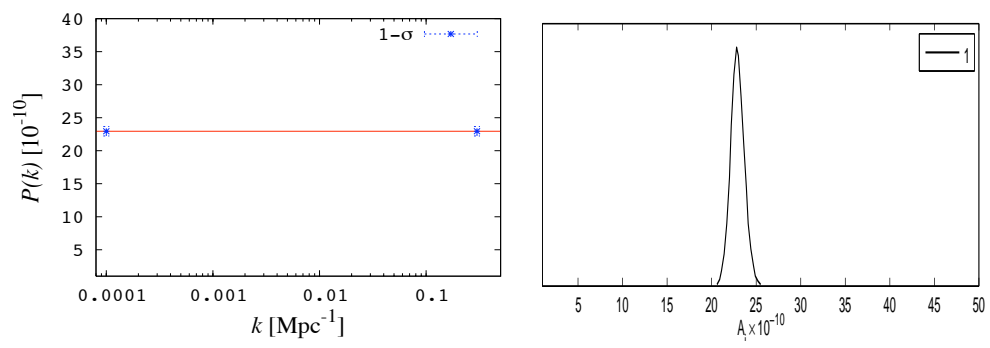

(b) $\mathcal{B}_{2,1}=+2.93 \pm 0.30$
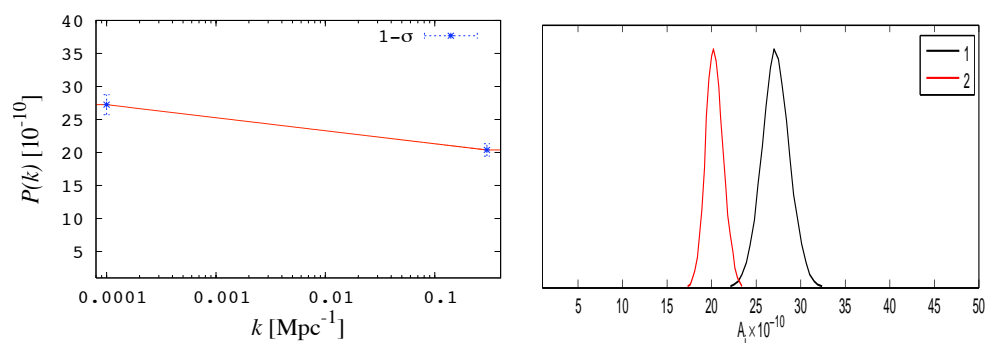

(c) $\mathcal{B}_{3,1}=+2.75 \pm 0.30$
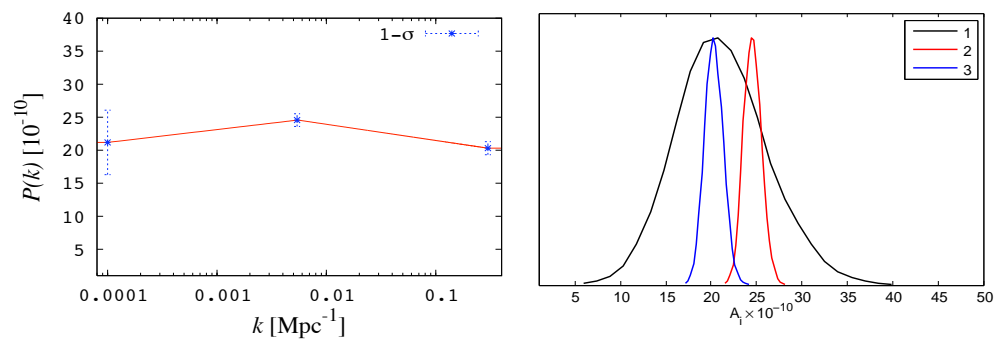

(d) $\mathcal{B}_{4,1}=+0.67 \pm 0.30$
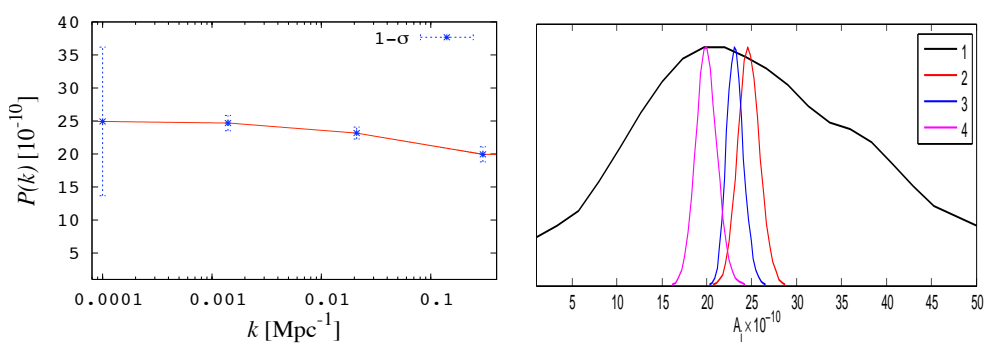

Figure 1. Left: Reconstruction of the primordial spectrum modelled as piecewise linear between nodes with fixed wavenumber $k_{i}$, along with mean amplitude values and their corresponding $1 \sigma$ error bars. On large scales the power spectrum is constrained by WMAP data, whereas at small scales ACT/LRG data yield tight constraints up to $k=0.3 \mathrm{Mpc}^{-1}$. Right: 1D marginalised posterior distribution of the amplitudes $A_{i}$ at each bin in each reconstruction. The top label in each panel denotes the associated Bayes factor respect to the base model (HZ) (a).

ing to the Akaike information criterion, the preferred model is given by a two-node spectrum.

To extract the global structure of the spectrum we have carried out a reconstruction process by placing nodes at particular positions in the $k$-space. However, to localise features 
in $k$-space, we may consider moving either back or forth the internal $k$-nodes until we find their optimal position; we reconsider this option in an improved method in the next Section.

\subsection{Power spectrum reconstruction II}

In Section 3.1 we reconstructed the primordial spectrum using a standard binning process: fix $k$-node positions and vary only the amplitudes. We now consider a reconstruction of the spectrum where the internal $k$-node positions vary, as well as their amplitudes.

\subsubsection{Running-like spectrum}

In order to look for deviations from the simple power-law model, we consider a model with two fixed $k$-nodes at sufficiently separated positions $\left[k_{\min }, k_{\max }\right]$, with varying amplitudes $\left[A_{\mathrm{s}, k_{\min }}, A_{\mathrm{s}, k_{\max }}\right]$, and place inside additional 'nodes' with the freedom to move around in both position $k_{i}$ and amplitude $A_{\mathrm{s}, k_{i}}$. Despite the simplicity of this approach, it covers a large variety of shapes for the primordial spectrum. The freedom of the position of the internal $k$-nodes allows us to localise the best position for a turn-over (if any) and the amplitudes are able to describe the global structure of the spectrum.

Analogously to Section 3.1, we have maintained the same priors for the spectral amplitude $A_{\mathrm{s}, k_{i}}=[1,50] \times 10^{-10}$, whereas on the $k$-position we restrict to the physical prior $\log k_{i}=\left[\log k_{\min }, \log k_{\max }\right]$. Hence, for this type of nodal-reconstruction the spectrum is described by

$$
\mathcal{P}_{\mathcal{R}}(k)= \begin{cases}A_{\mathrm{s}, k_{\min }} & k \leq k_{\min } \\ A_{\mathrm{s}, k_{i}} & k_{\min }<k_{i}<k_{i+1}<k_{\max } \\ A_{\mathrm{s}, k_{\max }} & k \geq k_{\max }\end{cases}
$$

and with linear interpolation for $k_{\min } \leq k_{i} \leq k_{\max }$.

The internal nodes generalise the spectral running by allowing slight variations in the interpolated slopes between external nodes. Figure 2 illustrates the reconstruction of the shape of the primordial spectrum from the mean posterior estimates - with $1 \sigma$ error bars on the amplitudes - (left), along with the 1D marginalised posterior distributions on the parameters used to describe the spectrum (right). On large scales, the reconstructed shape of the one-internal-node model $\left(k_{1}\right)$ resembles a similar spectrum to that obtained in Figure 1 (c), but now the probability distribution suggests a preferred turn-over position localised at the largest scales. A similar turn-over has also been identified using principle component analysis [17]. In the two and three-internal-nodes cases, it is interesting to note that at small scales the marginalised posterior peaks at scales where the combined WMAP/ACT constraints are improved; at these scales $\left(0.1<k\left[\mathrm{Mpc}^{-1}\right]<0.14\right)$ CMB data now considerably overlap with measurements from SDSS DR7 LRG. This reduced power at small scales might be identified as a feature produced from a phase transition in the early universe [5]. Both, the two and three-internal models (middle and bottom panel of Figure 2), present a similar behaviour on the reconstructed spectra, also seen on the marginalised posterior distributions.

\subsubsection{Cut-off and Broken spectra}

For completeness, we consider the possible existence of a large-scale cut-off on the primordial spectrum. A possible motivation to consider this model has been discussed for instance by 
$\left(k_{1}\right) \mathcal{B}_{k_{1}, 1}=+4.26 \pm 0.30$
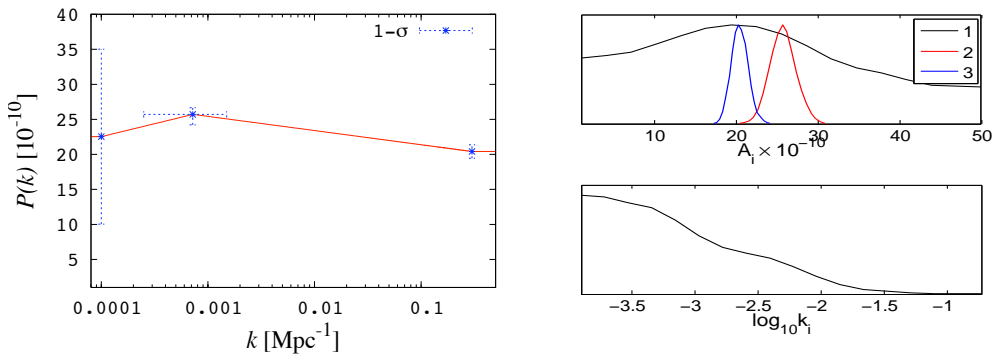

$\left(k_{2}\right) \mathcal{B}_{k_{2}, 1}=+3.73 \pm 0.30$
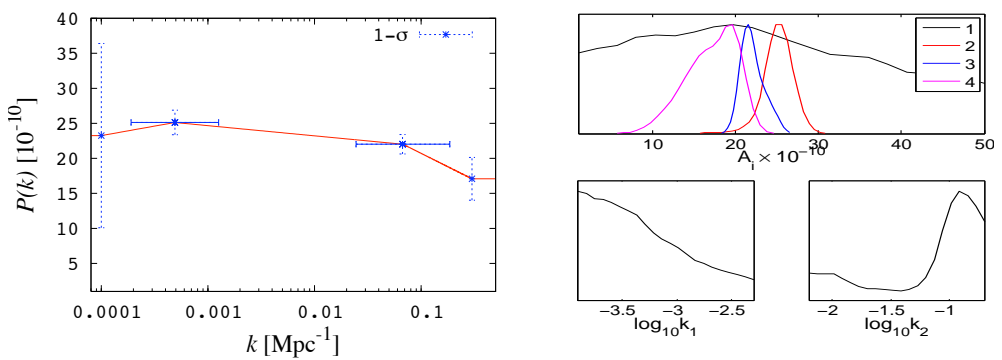

$\left(k_{3}\right) \mathcal{B}_{k_{3}, 1}=+3.49 \pm 0.30$
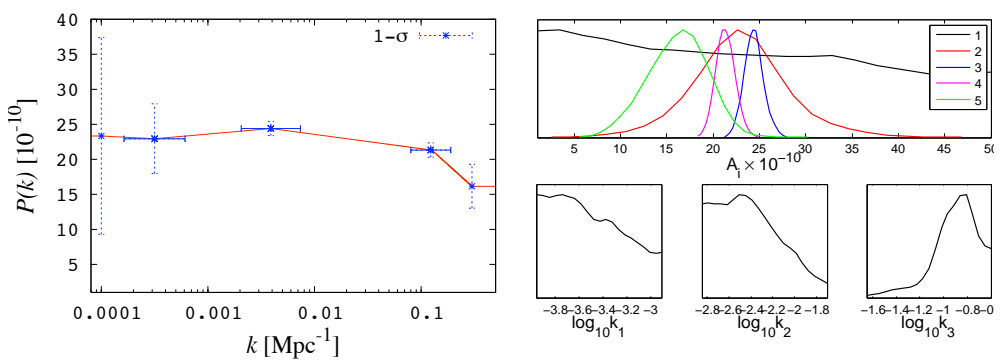

Figure 2. Reconstruction of the primordial spectrum letting one internal $k$-node (top) and two internal $k$-nodes (bottom) move freely in both amplitude $A_{i}$ and position $k_{i}$. The right panel corresponds to the 1D marginalised posterior distribution of the amplitudes and $k$-node position in each reconstruction. The top label in each panel denotes the associated Bayes factor with respect to the base model (HZ) shown in Figure 1 (a).

[27]. In order to perform the reconstruction for this particular case we fix an extremal node at $k_{\max }$ with varying amplitude $A_{\mathrm{s}, k_{\max }}$ and let the cut-off scale $k_{c}$ vary across the prior $\left[k_{\min }, k_{\max }\right]$ as well as its amplitude $A_{\mathrm{s}, c}$. The form of the spectrum is described as follows:

$$
\mathcal{P}_{\mathcal{R}}(k)= \begin{cases}0 & k \leq k_{c} \\ A_{\mathrm{s}, c} & k_{c}<k<k_{\max } \\ A_{\mathrm{s}, \max } & k \geq k_{\max }\end{cases}
$$

and with linear interpolation for $k_{c}<k<k_{\max }$.

Also, we consider a broken spectrum which might have been produced from phase transitions in the early universe. A similar broken spectrum, motivated by double or multiple field 
$\left(k_{c}\right) \mathcal{B}_{k_{c}, 1}=+2.98 \pm 0.30$
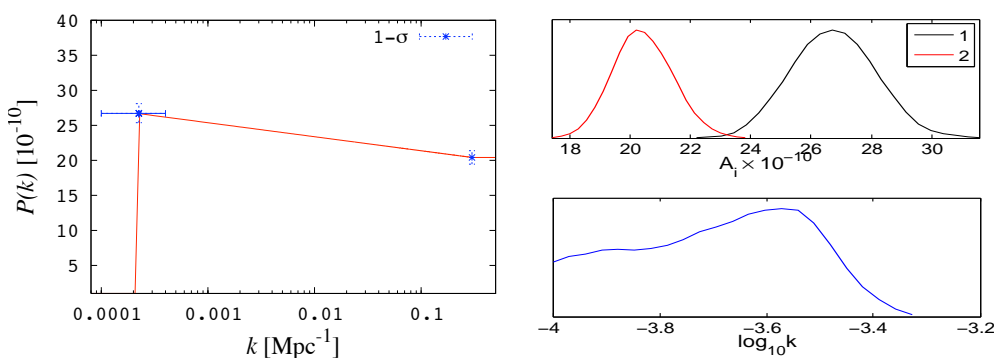

$\left(k_{b}\right) \mathcal{B}_{k_{b}, 1}=+2.38 \pm 0.30$
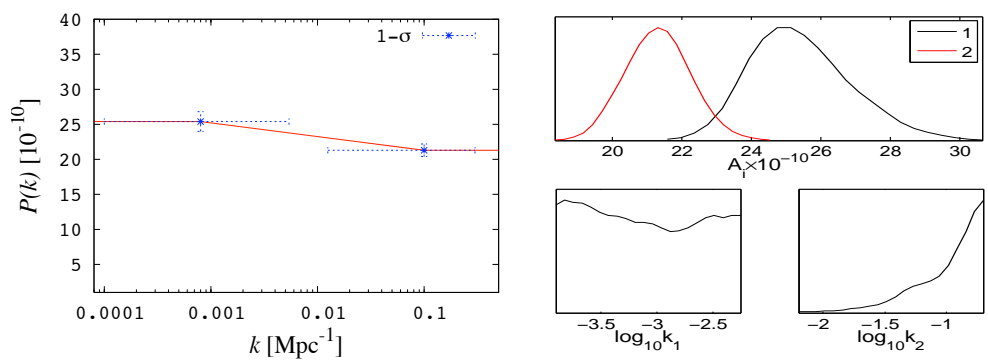

Figure 3. Reconstruction of the large scale cut-off spectrum (top) and broken spectrum (bottom); their corresponding 1D marginalised posterior distribution of the amplitudes $A_{\mathrm{i}}$ and node positions $k_{i}$ in each reconstruction (right). The top label in each panel denotes the associated Bayes factor with respect to the base model (HZ) shown in Figure 1 (a).

inflation, has been considered by $[5,7]$. This spectrum is obtained by placing two nodes $k_{1}$ and $k_{2}$ within $\left[k_{\min }, k_{\max }\right]$, and letting them move freely in amplitude $A_{\mathrm{s}, k_{i}}$ and $k$-position, such that

$$
\mathcal{P}_{\mathcal{R}}(k)= \begin{cases}A_{\mathrm{s}, k_{1}} & k<k_{1} \\ A_{\mathrm{s}, k_{2}} & k \geq k_{2}\end{cases}
$$

and with linear interpolation for $\quad k_{1}<k_{2}$.

The reconstruction of the cut-off and broken primordial spectra along with $1 \sigma$ limits of the marginalised distributions are shown in the left panels of Figure 3. Their corresponding posterior distribution in each parameter used to describe the spectra are illustrated in the right panels. The obtained best-fit parameters for the cut-off spectrum (top), show a preferred scale at which the power drops to zero with an upper $\operatorname{limit} \log _{10} k_{c}<-3.45$ at $95 \%$ C.L. Our constraints on $k_{c}$ also show a significant likelihood at large scales, thereby disfavouring the presence of an abrupt cut-off. With respect to the broken model (bottom), on the other hand, the best fit parameters indeed predict a break in the primordial spectrum, located approximately at $\log _{10} k \simeq-2.2$. That could be an indicative of the existence of a phase transition, and it is similarly obtained in the two and three-internal-nodes models shown in Figure 2.

In this section we have considered three types of spectra with different features: turnover, large scale cut-off and broken spectrum (Figures 2-3). In each figure we have included 
the Bayes factor compared to the base model (HZ). According to the Jeffreys guideline the one-internal-node spectrum, shown in the top panel of Figure 2, is significantly preferred over the cut-off and broken spectrum models, $\mathcal{B}_{k_{1}, k_{c}}=+1.28 \pm 0.30$ and $\mathcal{B}_{k_{1}, k_{b}}=+1.88 \pm 0.30$ respectively. Even though the model with one-internal node is described by four parameters, when it is compared to the Harrison-Zel'dovich spectrum (with only one parameter), the Bayes factor $\mathcal{B}_{k_{1}, 1}=+4.26 \pm 0.30$ shows that the presence of a turn-over is strongly favoured by current cosmological information and significantly so when compared to the tilted spectrum (with two parameters) $\mathcal{B}_{k_{1}, 2}=+1.33 \pm 0.30$, see Figure 1 . Therefore, the presence of a turn-over in $\mathcal{P}_{\mathcal{R}}(k)$ plays an important role in explaining current observations. Notice that, in the bottom panel of Figure 2 the Bayesian evidence has dropped off, hence the reason we have stopped the addition of nodes in the reconstruction process.

\subsection{Power-law and running spectra}

We have considered, so far, a $\mathcal{P}_{\mathcal{R}}(k)$ shape reconstructed directly from data. For comparison we include the standard approach by assuming a power-law parameterisation in terms of a spectral amplitude $A_{\mathrm{s}}$ and a spectral index or tilt parameter $n_{\mathrm{s}}$ :

$$
\mathcal{P}_{\mathcal{R}}(k)=A_{\mathrm{s}}\left(\frac{k}{k_{0}}\right)^{n_{\mathrm{s}}-1},
$$

where the spectral index is expected to be close to unity; $k_{0}$ denotes the scale at which the tilted spectrum pivots, fixed to $k_{0}=0.002 \mathrm{Mpc}^{-1}$. We again assume the prior $A_{\mathrm{s}}=$ $[1,50] \times 10^{-10}$ on the amplitude, together with the conservative prior $n_{\mathrm{s}}=[0.7,1.2]$ on the spectral index. We find a mean value of $n_{\mathrm{s}}=0.963 \pm 0.011$ which confirms that our constraints are in good agreement with results from [1-3]. As a further extension we consider possible deviations from power-law by allowing the spectral index to vary as a function of scale $n_{\mathrm{s}}(k)$. Then the primordial spectrum becomes

$$
\mathcal{P}_{\mathcal{R}}(k)=A_{\mathrm{S}}\left(\frac{k}{k_{0}}\right)^{n_{\mathrm{s}}-1+\frac{1}{2} \ln \left(\frac{k}{k_{0}}\right) n_{\mathrm{run}}},
$$

where $n_{\text {run }}$ is termed the running parameter and is expected to be $n_{\text {run }} \approx 0$ for standard inflationary models. In order to minimise the correlation between $n_{\mathrm{s}}$ and $n_{\text {run }}$ we have considered a pivot scale of $k_{0}=0.015 \mathrm{Mpc}^{-1}$, as pointed out by [28]. We use the same priors as above on $A_{\mathrm{s}}$ and $n_{\mathrm{s}}$, and the conservative prior $n_{\text {run }}=[-0.3,0.3]$ on the running parameter. From the combined dataset we find the marginalised posteriors show a preference for a negative running parameter $n_{\text {run }}=-0.026 \pm 0.015$ and $n_{\mathrm{s}}=0.968 \pm 0.011$ for the spectral index, as expected by [1-3]. Figure 4 shows the marginalised posterior distributions for the parameters used to describe $\mathcal{P}_{\mathcal{R}}(k)$ and the obtained spectrum from mean posterior estimates of a simple tilted parameterisation with $n_{\mathrm{S}}$ (left) and including the running parameter $n_{\text {run }}$ (right) respectively. In each panel we have included the Bayes factor compared to the base model (HZ).

According to the Jeffreys guideline, present observations significantly prefer a simple tilted model when compared to a model which includes a running parameter by a factor $\mathcal{B}_{n_{\mathrm{s}}, n_{\text {run }}}=+1.19 \pm 0.30$. Similarly, a tilted spectrum is strongly preferred when compared to the HZ model: $\mathcal{B}_{n_{\mathrm{s}}, 1}=+3.25 \pm 0.30$. We also confirm the agreement between the simple tilted model and the two-fixed-noded spectrum through its Bayes factor, shown in Figure 1 (b). An important point to emphasise is that the simple tilt and running model present 


$$
\left(n_{\mathrm{s}}\right) \mathcal{B}_{n_{\mathrm{s}}, 1}=+3.25 \pm 0.30
$$
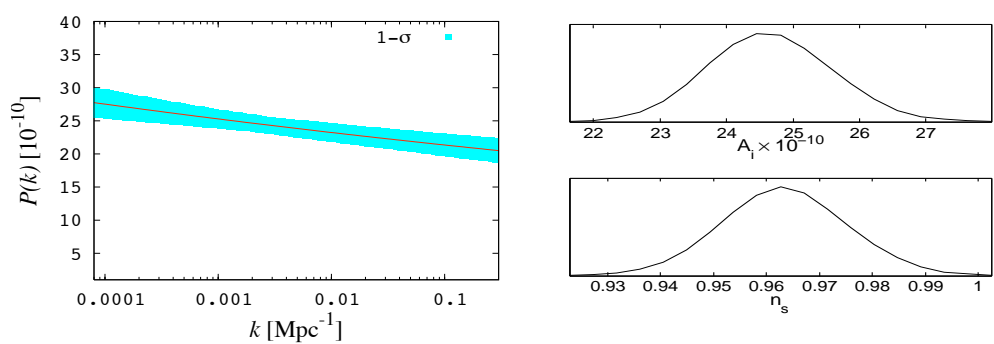

$$
\left(n_{\text {run }}\right) \mathcal{B}_{n_{\text {run }, 1}}=+2.06 \pm 0.30
$$
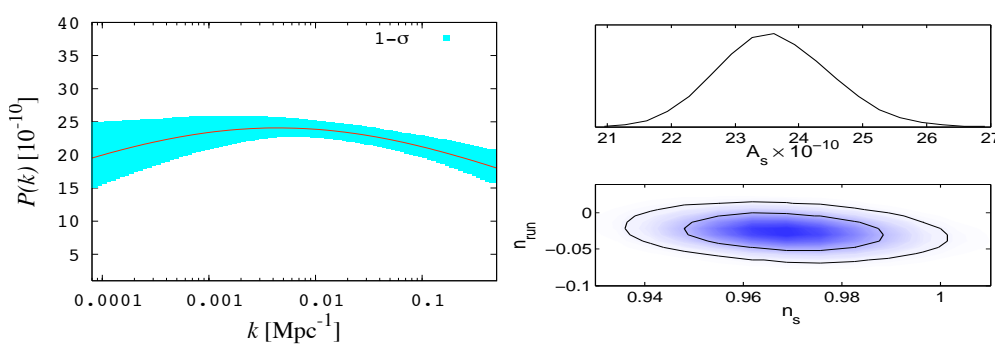

Figure 4. Reconstruction of the primordial spectrum assuming a simple tilted parameterisation with $n_{\mathrm{s}}$ (top) and including the running parameter $n_{\text {run }}$ (bottom); the coloured region denotes $1 \sigma$ error bands on the reconstruction. Right: marginalised 1D and 2D probability posterior distributions for the power spectrum parameters; 2D constraints are plotted with $1 \sigma$ and $2 \sigma$ confidence contours. The top label in each panel denotes the associated Bayes factor with respect to the base model (HZ) shown in Figure 1 (a).

a significantly and strongly disfavoured Bayes factor, $\mathcal{B}_{n_{\mathrm{s}}, k_{1}}=-1.01 \pm 0.30, \mathcal{B}_{n_{\text {run }}, k_{1}}=$ $-2.20 \pm 0.30$, compared to the reconstructed one-internal-node spectrum shown in Figure 2. Thus a simple power-law parameterisation seems to be not enough to describe current cosmological observations, hence slight deviations of it should be taken into account.

\subsection{Modified Power-law spectrum}

We have observed that models which present a turn-over at large scales are slightly preferred by the evidence. Based on this observation, we suggest the following phenomenological shape for the primordial power spectrum:

$$
\mathcal{P}_{\mathcal{R}}(k)=A_{\mathrm{s}} \frac{\left(\frac{k}{k_{\mathrm{v}}}\right)^{n_{\mathrm{v}}}}{\frac{k}{k_{\mathrm{v}}}+1} .
$$

In this particular parameterisation, assuming $n_{\mathrm{v}}<1$, the parameter $k_{\mathrm{v}}$ determines the transition between a standard power-law model with red tilt $\left(k \gg k_{\mathrm{v}}\right)$ to a blue tilt model $\left(k \ll k_{\mathrm{v}}\right)$ :

$$
\mathcal{P}_{\mathcal{R}}(k)=A_{\mathrm{S}} \begin{cases}\left(\frac{k}{k_{\mathrm{v}}}\right)^{n_{\mathrm{v}}-1} & k \gg k_{\mathrm{v}}, \\ \left(\frac{k}{k_{\mathrm{v}}}\right)^{n_{\mathrm{v}}} & k \ll k_{\mathrm{v}},\end{cases}
$$




$$
\left(n_{\mathrm{v}}\right) \mathcal{B}_{n_{\mathrm{v}}, 1}=+4.65 \pm 0.30
$$
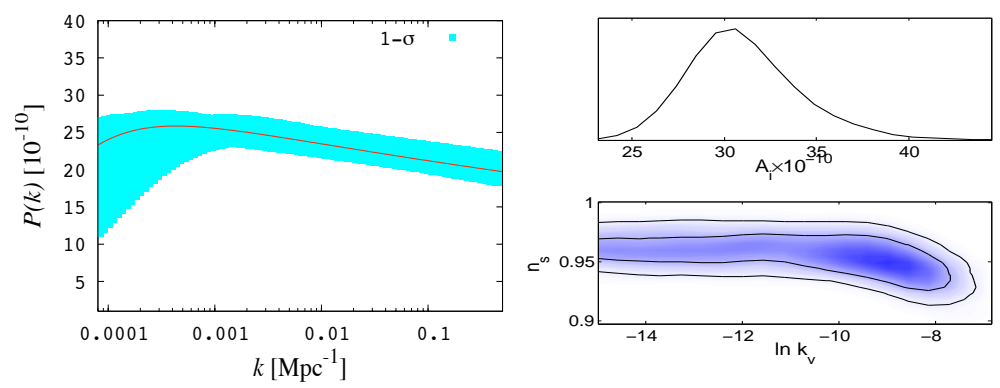

Figure 5. Reconstruction of the primordial spectrum from mean posterior estimates of the modified power-law parameterisation, along with $1 \sigma$ error bands (left). Right: marginalised $1 \mathrm{D}$ and 2D probability posterior distributions for the power spectrum parameters; $2 \mathrm{D}$ constraints are plotted with $1 \sigma$ and $2 \sigma$ confidence contours. The top label in each panel denotes the associated Bayes factor with respect to the base model (HZ) shown in Figure 1 (a).

where the prior on $n_{\mathrm{v}}$ is similarly chosen to the spectral index $n_{\mathrm{s}}$ in the power-law parameterisation: $n_{\mathrm{v}}=[0.7,1.2]$. We expect the constraints on the parameter $k_{\mathrm{v}}$ are mainly located on large scales, hence, for this extra-parameter we consider the following flat prior $\ln k_{\mathrm{v}}=[-15,-5]$. The reconstruction of the shape of this spectrum along with the posterior distribution in each parameter are shown in Figure 5. We observe the constraints for the new tilt-parameter $n_{\mathrm{v}}=0.955 \pm 0.014$ are similar to those obtained from the power-law models, where the scale-invariant spectrum is ruled out at a high confidence level and the spectrum exhibits a red tilt at small scales. The marginalised posterior probability on $k_{\mathrm{v}}$ shows the existence of a blue-tilted spectrum on large scales $\ln k_{\mathrm{v}}<-8.1$ at $95 \%$ C.L. Hence, the global shape for this spectrum presents a slight running behaviour with reduced power at both large and small scales, compared to the simple tilt parameterisation.

The modified power-law parameterisation decisively rules out the HZ, since the Bayes factor between the models is $\mathcal{B}_{n_{\mathrm{v}}, 1}=+4.65 \pm 0.30$. Moreover, even though the modified power-law model has an extra parameter compared to the simple power-law model, the data significantly prefer it with $\mathcal{B}_{n_{\mathrm{v}}, n_{\mathrm{s}}}=+1.40 \pm 0.30$.

\subsection{Lasenby \& Doran spectrum}

The Lasenby and Doran model (LD, [6]) is based on the restriction of the total conformal time available in the entire history of a closed Universe. The primordial spectrum derived from this model naturally incorporates an exponential cut-off on large scales which might provide a possible explanation for the lower-than-expected CMB power spectrum at low multipoles. On small scales, the relationship between $\mathcal{P}_{\mathcal{R}}^{1 / 2}(k)$ and $\ln k$ is linear, thus predicting a reduced power at large $k$ as compared to a simple tilted spectrum (for which $\ln \mathcal{P}_{\mathcal{R}}^{1 / 2}(k)$ versus $\ln k$ is linear). For further details about the LD model see, for instance [29, 30]. In this model, the resultant primordial spectrum from the inflationary phase depends upon just two parameters $\left\{b_{0}, b_{4}\right\}$. The parameter $b_{0}$ is mainly restricted by the number of $e$-folds $N \approx 2 \pi b_{0}^{2} \approx 50$, whereas $b_{4}$ controls the initial curvature and must be negative and such that the dimensionless combination $\left|b_{4}\right| \mu^{-4 / 3}$ is of order unity. Here $\mu$ is the mass of the scalar field, and this 


$$
\left(n_{\mathrm{LD}}\right) \mathcal{B}_{\mathrm{LD}, 1}=+4.94 \pm 0.30
$$
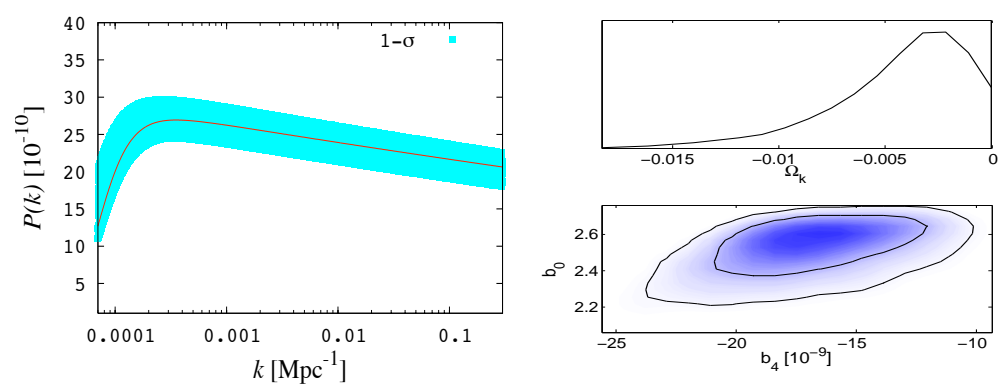

Figure 6. Reconstruction of the primordial spectrum assuming a Lasenby \& Doran model along with $1 \sigma$ error bands (left). Right: marginalised 1D and 2D probability posterior distributions for the power spectrum parameters; $2 \mathrm{D}$ constraints are plotted with $1 \sigma$ and $2 \sigma$ confidence contours. The top label on the panel denotes the associated Bayes factor with respect to the base model (HZ) shown in Figure 1 (a).

along with $b_{0}$ and $b_{4}$ control the magnitude of the inflaton field, and how long the inflationary period lasts. To compute the LD spectrum we refer our analysis to [19]. We have also chosen the priors based on the same paper: $\Omega_{k}=\left[-0.05,10^{-4}\right], b_{0}=[1,4], b_{4}=[-30,-1] \times 10^{-9}$. Figure 6 shows the reconstructed shape of the primordial spectrum along with the posterior distribution in each additional parameter for this model; the constraints on the present hubble parameter are $H_{0}=69.4 \pm 1.4$, whereas the number of $e$-folds is $N=50.6 \pm 4.3$. From the top label of Figure 6, we observe the LD model is significantly preferred over the simple power-law parameterisation with a Bayes factor of $\mathcal{B}_{\mathrm{LD}, n_{\mathrm{s}}}=+1.69 \pm 0.30$ and decisive when compared to the $\mathrm{HZ}$ spectrcum: $\mathcal{B}_{\mathrm{LD}, 1}=+4.94 \pm 0.30$.

Indeed, the LD model has the largest evidence of all the models investigated, followed closely by the modified power-law spectrum. It should be noted, however, that the latter was constructed specifically to exhibit a turn-over on large scales, having already found that the data prefer such a feature. A fairer comparison would be between the LD model and the third most favoured model, namely the one-internal-node linear-interpolation model described in Section 3.2.1, since both of these models were proposed a priori. To this end, and as a check on our analysis, we use the best-fit LD model (shown in Figure 6) as the input spectrum to simulate an idealised CMB observation containing only cosmic-variance-limited noise. Figure 7 (left panel) shows the resulting CMB temperature spectrum. We then analysed these simulated data using the one-internal-node linear interpolation model to reconstruct the primordial power spectrum. Figure 7 (right panel) shows the resulting reconstruction (dotted line), which recovers well the shape of the input LD spectrum (solid line), except on the very largest scales, where there is little information in the simulated CMB data. Moreover, the reconstructed spectrum has a similar shape to the one obtained from real data using the one-internal-node model (see Figure 2). We may therefore understand the higher evidence for the LD model spectrum as resulting from its similar quality fit to the data, but requiring fewer free parameters than the one-internal-node linear-interpolation model.

Finally, we note that, in the node-based reconstruction, the use of linear interpola- 

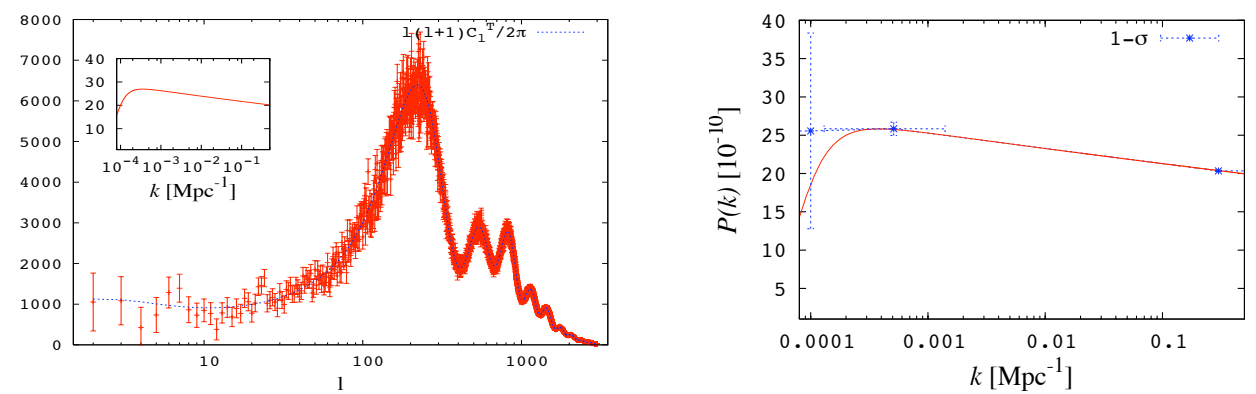

Figure 7. Reconstruction of the Lasenby \& Doran primordial spectrum based on the binning format with linear interpolation described in Section 3.2.1. We have assumed an idealised CMB spectrum with limitation only due to cosmic variance (left). Right panel shows the reconstructed spectrum in the binning format together with the LD input spectrum (solid line).

tion between the nodes may seem crude. It is straightforward to generalise the node-based approach to more sophisticated interpolation schemes, but this may not always yield better results. In Appendix A, we illustrate this point by reanalysing the simulated CMB data using a cubic spline interpolation through the nodes, thus allowing one to reconstruct a smooth shape for the primordial spectrum, but one that is less satisfactory than that obtained using linear interpolation.

\section{Discussion and Conclusions}

In this paper we have attempted to fit an optimal degree of structure for the primordial power spectrum of curvature perturbations using Bayesian model selection as our discriminating criterion. We have modeled the spectrum as a linear interpolation between a set of 'nodes' with varying amplitude and $k$-position. We have also explored different parameterisations of the primordial spectrum which include: a power-law parameterisation with both tilt and running parameter, a modified power-law spectrum and the Lasenby \& Doran (LD) model.

All the considered models have in common the standard cosmological parameters: $\Omega_{\mathrm{b}} h^{2}$, $\Omega_{\mathrm{DM}} h^{2}, \theta, \tau$, as well as the secondary parameters: $A_{S Z}, A_{p}, A_{c}$. Thus, priors on these parameters remained the same in each model. The best-fit values for these standard parameters are consistent with those obtained using the concordance 6-parameter model with power-law primordial spectrum. We show, in Figure 8, 1D marginalised posterior distributions for the cosmological parameters of each of the preferred models. We observe that the values of the standard parameters remain well constrained despite the addition of extra freedom on the shape of the primordial spectrum, although the constraints resulting from the HZ spectrum clearly differ from the others. Note also that the constraints on the parameters corresponding to the LD model are slightly tighter than the rest of the models.

We have considered wide-enough priors in our analysis, such that they do not interfere with the inferred parameter values. We used priors on the amplitudes of $A_{i}=[1,50] \times 10^{-10}$ and on spectral indices of $n_{\mathrm{s}}=n_{\mathrm{v}}=[0.7,1.2]$, while the parameters describing the $k$-space 

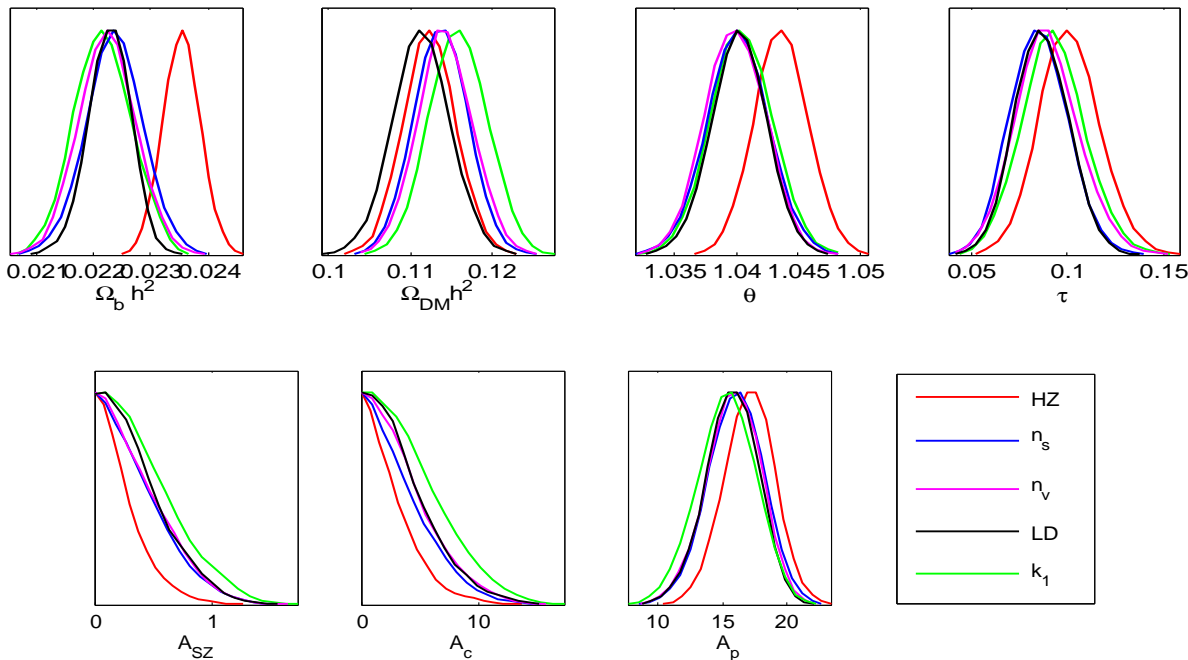

Figure 8. 1D marginalised posterior distributions for the primary (top) and secondary (bottom) cosmological parameters, for each corresponding model listed on the bottom-right box.

have physical priors restricted by $\left[k_{\min }, k_{\max }\right]$. We also compute the Bayesian evidence for a wider prior range of $n_{\mathrm{s}}=n_{\mathrm{v}}=[0.5,1.5]$ and $b_{4}=[-50,-1] \times 10^{-9}$, to illustrate the robustness of a model over small variations of the prior range:

$$
\begin{array}{ll}
\mathcal{B}_{n_{\mathrm{s}}, 1}=+2.25 \pm 0.30 & \text { (wide priors) } \\
\mathcal{B}_{n_{\mathrm{v}}, 1}=+4.24 \pm 0.30 & \text { (wide priors) } \\
\mathcal{B}_{\mathrm{LD}, 1}=+4.47 \pm 0.30 & \text { (wide priors) }
\end{array}
$$

We observe that even when wider priors are considered, the HZ model is strongly disfavoured when compared to $n_{\mathrm{v}}$ and LD models. Similarly, the simple tilted model is still significantly disfavoured.

To summarise the analysis, in Figure 9 we plot the reconstructed spectra for the preferred selected models together with their corresponding Bayesian evidence. It shows that the HZ spectrum is decisively excluded as a viable model to describe $\mathcal{P}_{\mathcal{R}}(k)$. The preferred model given current observations is provided by the LD model followed by a modified powerlaw version. We have found that the power-law parameterisation, including either cases $n_{\mathrm{s}}$ and $n_{\mathrm{s}}+n_{\text {run }}$, are both significantly disfavoured. The presence of a turn-over at large scales ${ }^{3}$ and the reduced power at small scales seem to provide an important contribution on choosing the best-fit model through its Bayesian evidence.

\footnotetext{
${ }^{3}$ At the largest scales, the addition of tensor perturbations might considerably affect the shape of the primordial spectrum. Hence, we consider this possibility in a more detailed future work.
} 


\begin{tabular}{cccc}
\hline \hline Model & $\mathrm{N}_{\text {par }}$ & $-2 \Delta \ln \mathcal{L}_{\max }$ & Bayes factor \\
\hline $\mathrm{HZ}$ & 8 & 0.0 & $\mathcal{B}_{1,1}=+0.0 \pm 0.3$ \\
$n_{\mathrm{s}}$ & 9 & -8.6 & $\mathcal{B}_{n_{\mathrm{s}}, 1}=+3.3 \pm 0.3$ \\
$n_{\mathrm{v}}$ & 10 & -9.4 & $\mathcal{B}_{n_{\mathrm{v}}, 1}=+4.7 \pm 0.3$ \\
$\mathrm{LD}$ & 10 & -9.4 & $\mathcal{B}_{\mathrm{LD}, 1}=+4.9 \pm 0.3$ \\
$k_{1}$ & 11 & -9.1 & $\mathcal{B}_{k_{1}, 1}=+4.3 \pm 0.3$ \\
\hline \hline
\end{tabular}

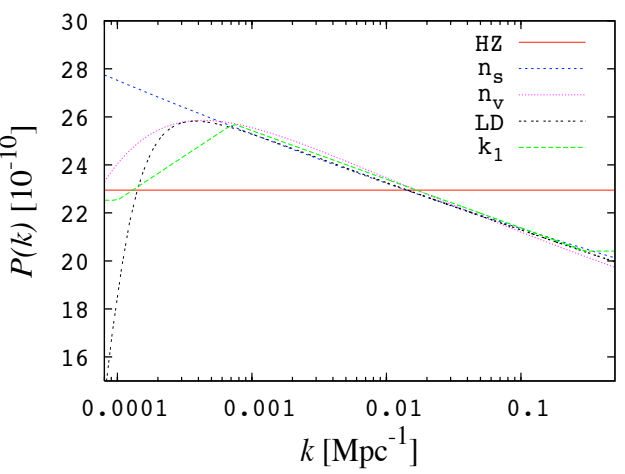

Figure 9. Comparison of the primordial power spectra for the preferred models along with their Bayesian evidence. We also include the maximum likelihood $\mathcal{L}_{\max }$ for a model with number of parameters $\mathrm{N}_{\mathrm{par}}$. Each Bayes factor is compared respect to the one-node model (HZ).

\section{Acknowledgments}

This work was carried out largely on the Cambridge High Performance Computing cluster, DARWIN. JAV is supported by CONACYT México.

\section{References}

[1] Komatsu E. et al. Seven-Year Wilkinson Microwave Anisotropy Probe (WMAP) Observations: Cosmological Interpretation. The Astrophysical Journal Supplement Series, 192(2):18, 2011. [arXiv:1001.4538].

[2] Dunkley J. et al. The Atacama Cosmology Telescope: Cosmological Parameters from the 2008 Power Spectra. [arXiv:1009.0866].

[3] Keisler R. et al. A Measurement of the Damping Tail of the Cosmic Microwave Background Power Spectrum with the South Pole Telescope. [arXiv:1105.3182].

[4] Efstathiou G. Is the Low Cosmic Microwave Background Quadrupole a Signature of Spatial Curvature? Monthly Notices of the Royal Astronomical Society, 343(4):L95-L98, 2003. [arXiv:0303127].

[5] Barriga J., Gaztanaga E., Santos M.G., and Sarkar S. On the APM power spectrum and the CMB anisotropy: Evidence for a phase transition during inflation? Monthly Notices of the Royal Astronomical Society, 324(4):977-987, 2001. [arXiv:0011398].

[6] Lasenby A. and Doran C. Closed Universes, de Sitter Space, and Inflation. Phys. Rev. D, 71:063502, Mar 2005. [arXiv:0307311].

[7] Bridle S. L., Lewis A. M., Weller J., and Efstathiou G. Reconstructing the primordial power spectrum. Monthly Notices of the Royal Astronomical Society, 342(4):L72-L78, 2003. [arXiv:0302306].

[8] Mukherjee P. and Wang Y. Primordial Power Spectrum Reconstruction. Journal of Cosmology and astroparticle Physics, 2005(12):007, 2005. [arXiv:0502136].

[9] Tocchini-Valentini D., Hoffman Y., and Silk J. Non-parametric reconstruction of the primordial power spectrum at horizon scales from WMAP data. Monthly Notices of the Royal Astronomical Society, 367(3):1095-1102, 2006. [arXiv:0509478]. 
[10] Hamann J., Shafieloo A., and Souradeep T. Features in the primordial power spectrum? A frequentist analysis. Journal of Cosmology and Astroparticle Physics, 2010(04):010, 2010. [arXiv:0912.2728].

[11] Ichiki K., Nagata R., and Yokoyama J. Cosmic Discordance: Detection of a modulation in the primordial fluctuation spectrum. Phys. Rev. D, 81:083010, Apr 2010. [arXiv:0911.5108].

[12] Bridges M., Lasenby A. N., and Hobson M. P. A bayesian analysis of the primordial power spectrum. Monthly Notices of the Royal Astronomical Society, 369(3):1123-1130, 2006. [arXiv:0511573].

[13] Bridges M., Lasenby A. N., and Hobson M. P. WMAP 3-yr primordial power spectrum. Monthly Notices of the Royal Astronomical Society, 381(1):68-74, 2007. [arXiv:0607404].

[14] Hlozek R. el al. The Atacama Cosmology Telescope: a measurement of the primordial power spectrum. [arXiv:1105.4887].

[15] Guo Z. K., Schwarz D. J., and Zhang Y. Z. Reconstruction of the primordial power spectrum from CMB data. Journal of Cosmology and Astroparticle Physics, 2011(08):031, 2011. [arXiv:1105.5916].

[16] Bridges M., Feroz F., Hobson M. P., and Lasenby A. N.. Bayesian optimal reconstruction of the primordial power spectrum. Monthly Notices of the Royal Astronomical Society, 400(2):1075-1084, 2009. [arXiv:0812.3541].

[17] Zhang Y. Z. and Guo Z. K. Uncorrelated estimates of the primordial power spectrum. [arXiv:1109.0067].

[18] Peiris H. V. and Verde L. The Shape of the Primordial Power Spectrum: A Last Stand Before Planck. Phys. Rev. D, 81:021302, Jan 2010. [arXiv:0912.0268].

[19] Vazquez J. A., Lasenby A. N., Bridges M. and Hobson M. P. A Bayesian study of the primordial power spectrum from a novel closed universe model. [arXiv:1103.4619].

[20] Amanullah R. et al. Spectra and Hubble Space Telescope Light Curves of Six Type Ia Supernovae at $0.511<z<1.12$ and the Union2 Compilation. The Astrophysical Journal, 716(1):712, 2010. [arXiv:1004.1711].

[21] Reid B.A. et al. Cosmological Constraints from the Clustering of the Sloan Digital Sky Survey DR7 Luminous Red Galaxies. Monthly Notices of the Royal Astronomical Society, 404(1):60-85, 2010. [arXiv:0907.1659].

[22] Riess A. G. et al. A Redetermination of the Hubble Constant with the Hubble Space Telescope from a Differential Distance Ladder. The Astrophysical Journal, 699(1):539, 2009. [arXiv:0905.0695].

[23] Lewis A., Challinor A., and Lasenby A. Efficient Computation of Cosmic Microwave Background Anisotropies in closed FRW models. The Astrophysical Journal, 538(2):473, 2000. [arXiv:9911177].

[24] Lewis A. and Bridle S. Cosmological Parameters from CMB and other data: A Monte Carlo approach. Phys. Rev. D, 66:103511, Nov 2002. [arXiv:0205436].

[25] Feroz F. and Hobson M. P. Multimodal nested sampling: an efficient and robust alternative to MCMC methods for astronomical data analyses. Monthly Notices of the Royal Astronomical Society, 384(2):449-463, 2008. [arXiv:0704.3704].

[26] Feroz F., Hobson M. P., and Bridges M. MultiNest: an efficient and robust Bayesian inference tool for cosmology and particle physics. Monthly Notices of the Royal Astronomical Society, 398(4):1601-1614, 2009. [arXiv:0809.3437].

[27] Contaldi C.R., Peloso M., Kofman L., and Linde A. Suppressing the lower Multipoles in the CMB anisotropies. Journal of Cosmology and Astroparticle Physics, 2003(07):002, 2003. 
[arXiv:0303636].

[28] Cortês M., Liddle A. R., and Mukherjee P. On what scale should inflationary observables be constrained? Phys. Rev. D, 75:083520, Apr 2007. [arXiv:0702170].

[29] Lasenby A. Conformal geometry and the universe. http ://www.mrao.cam.ac.uk/ clifford/publications/abstracts/anl_ima2002.html

[30] Lasenby A. and Doran C. Conformal Models of de Sitter Space, Initial Conditions for Inflation and the CMB. AIP Conference Proceedings, 736(1):53-70, 2004.

\section{A Cubic spline interpolated spectrum}

In this appendix we illustrate how the type of interpolation in our node-based approach can influence the reconstruction of the primordial spectrum. We use the same example shown in Figure 7 but now we use a cubic spline to interpolate through the $k$-nodes. From Figure 10 we note that the spectrum used as an input lies well outside the error bar on the reconstruction at low $k$-values. Therefore, the spline fails to recover the input spectrum correctly, contrary to the linear interpolation where the recovered spectrum, shown in Figure 7 , is more representative, with the true spectrum lying comfortably within the error-bars on the reconstruction at all $k$-values. This is mainly because a function with rapidly changing higher derivatives, such as the input primordial spectrum used here, is less accurately approximated by higher order polynomials. In particular, the requirement of continuous first and second derivatives, combined with the tight constraints at small and intermediate length scales, leads to a significant overestimation of the power at the less well constrained region at the largest scales. Hence, in this case, the linear interpolation describes the shape of the primordial spectrum better than the spline.

For comparison with the results presented earlier, we also use the cubic spline to perform similar node-based reconstructions of the spectrum to those shown in Figures 1 and 2. The low number of bins used to describe the global structure of the spectrum yield to similar shapes by using both interpolation methods. The reconstructed spectra for three and four bins, along with one and two internal $k$-nodes, are plotted in Figure 11 using the spline interpolation.
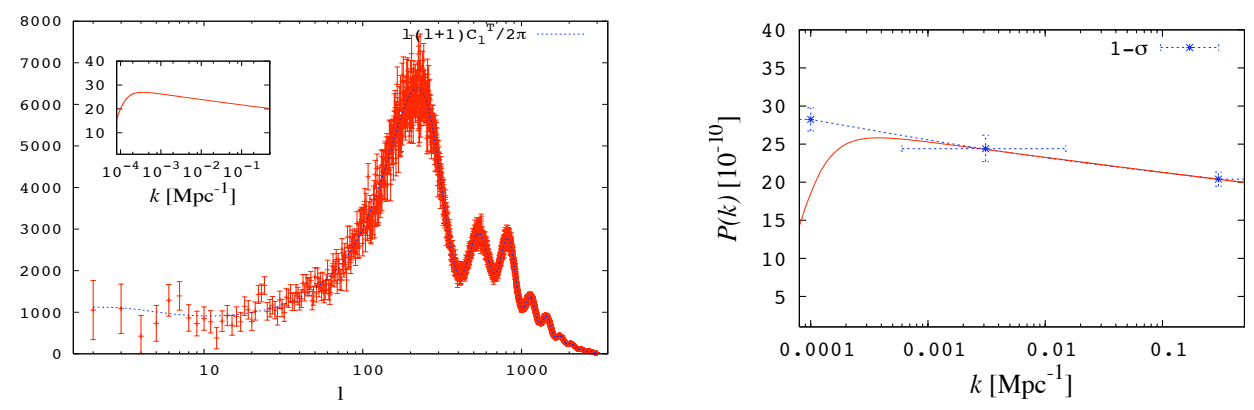

Figure 10. Reconstruction of the Lasenby \& Doran primordial spectrum based on the binning format with cubic spline described in Section 3.2.1. We have assumed an idealised CMB spectrum with limitation only due cosmic variance (left). Right panel shows the reconstructed spectrum in the binning format together with the LD input spectrum. 


$$
\mathcal{B}_{3,1}=+2.90 \pm 0.30
$$
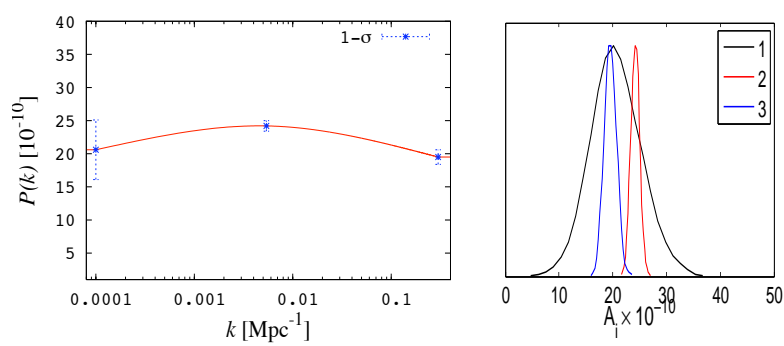

$\mathcal{B}_{k_{1}, 1}=+4.21 \pm 0.30$
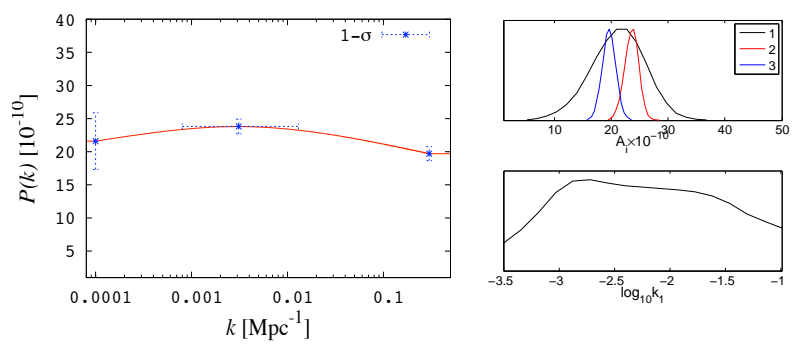

$\mathcal{B}_{k_{3}, 1}=+3.81 \pm 0.30$
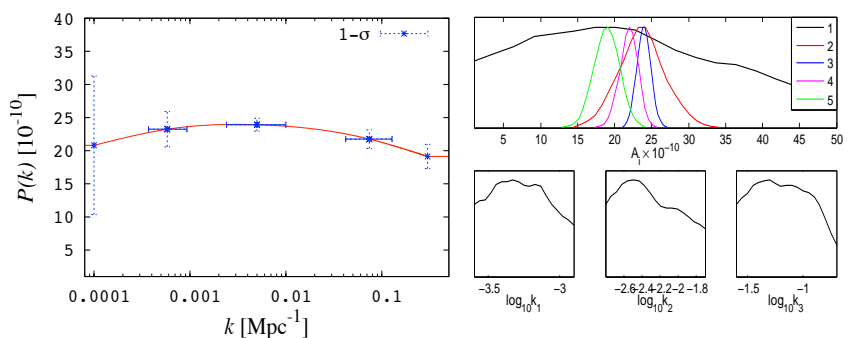

$\mathcal{B}_{4,1}=+1.62 \pm 0.30$
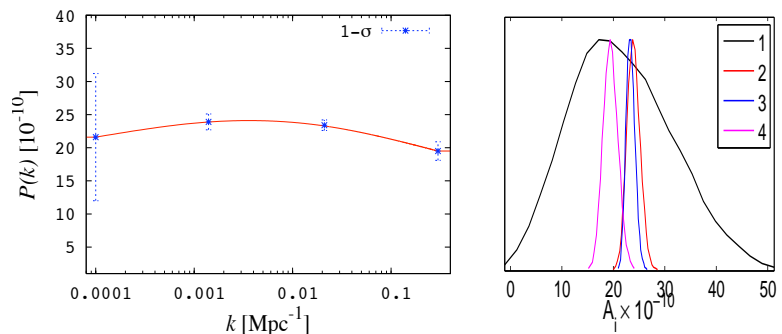

$\mathcal{B}_{k_{2}, 1}=+4.02 \pm 0.30$
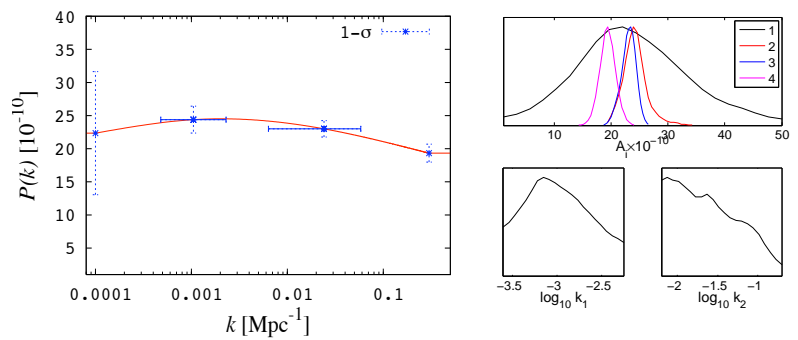

Figure 11. Reconstruction of the primordial spectrum using the cubic spline. Top panel resembles plots (b) and (c) shown in Figure 1, whereas bottom panel the reconstruction for the models shown in Figure 2. To the right of each reconstruction we plot the 1D marginalised posterior distribution of the amplitudes $A_{i}$ and $k$-node position $k_{i}$. The top label in each panel denotes the associated Bayes factor with respect to the base model (HZ) shown in Figure 1 (a). 